
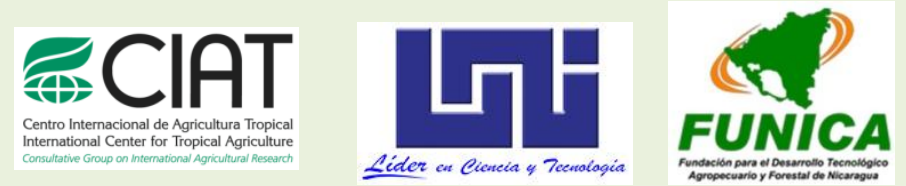

\title{
"Zonificación de Calidad de Café en la Zona Norte de Nicaragua, para Identificar Sitios Potenciales con Indicaciones Geográficas y/o Denominación de Origen (Resultados al I Trimestre 2012)"
}

Zelaya, Carlos*; Díaz, Alba**; Navarro, Sergio**; Dicovskiy, Luis**; García, Samuel ${ }^{* * *}$. ${ }^{*}$ Centro Internacional de Agricultura Tropical, CIAT, Nicaragua; ${ }^{* *}$ Universidad Nacional de Ingeniería (UNI, Sede Regional del Norte, Nicaragua); *** Pasante Centro Internacional de Agricultura Tropical CIAT, Nicaragua.

\section{Resumen}

La investigación "Zonificación de Calidad de Café en la Zona Norte de Nicaragua, para Identificar Sitios Potenciales con Indicaciones Geográficas y/o Denominación de Origen", contó con el apoyo técnico y financiero de FUNICA, y contempló la realización de estudios que demuestran la incidencia de los procesos de beneficiado y variables ambientales en la calidad de los perfiles de taza, de los departamentos de Nueva Segovia, Matagalpa y Jinotega de la región norte de Nicaragua. Para ello se realizó una recopilación 1200 datos de perfiles de taza utilizando el formato de catación que recomienda el SCAA para los atributos sensoriales. Los datos de catación fueron aportados por fuentes como ACEN, CISA y CONACAFE, los cuales se combinaron con mapas SIG de diferentes coberturas ambientales.

A partir de los análisis estadísticos y espaciales realizados a los datos de perfiles de taza y condiciones ambientales, se generaron los modelos y mapas que indican que los sitios que mantienen cada año elevados niveles de calidad de café son al menos cinco: Dipilto (Dipilto, Macuelizo y Mozonte), Las Sabanas (Las Sabanas, San Lucas, Pueblo Nuevo), San Juan de Río Coco (San Juan de Río Coco, Telpaneca) Jinotega (Jinotega, Datanli, el Diablo, Peñas Blancas) y San Rafael del Norte (San Rafael del Norte y San Sebastián de Yalí) identificándose como sitios potenciales para alcanzar una Indicación Geográfica y/o denominación de origen. El departamento de Nueva Segovia es el que presenta una tendencia hacia mejores atributos de calidad sobre Matagalpa y Jinotega, a partir de los análisis estadísticos multivariados. Los análisis confirman que hay un alto potencial para producir café de alta calidad en los sitios identificados.

Palabras clave: Denominación de Origen, Indicación Geográfica, Nichos ambientales, Calidad de café. 


\section{Introducción}

El proyecto de investigación "Zonificación de calidad de café en la zona Norte de Nicaragua" es coordinado por la Universidad Nacional de Ingeniería (UNI), con el apoyo técnico y financiero de la Fundación para el Desarrollo Tecnológico Agropecuario y Forestal de Nicaragua (FUNICA), y el apoyo técnico-científico (análisis espaciales) del Centro Internacional de Agricultura Tropical (CIAT). En este marco, para obtener los datos de catación, se estableció una alianza estratégica con CONACAFE, la Asociación de Cafés Especiales de Nicaragua (ACEN), CISA, Red del café, Escuela del Café, UCOSEMUN, y cooperativas de primer piso de la región norte como: UCANOR, CECOSPROCAE, COOMAPCOJ, ASOCAFEMAT, COOMPROCOM, UCAFE, ADEPROFOCA, CCAJ, APRODER, NUEVA ESPERANZA, ASOPANS, ASODAM, TEPEYAC, FLOR DE PINO Y FLOR DE CAFÉ. También es parte de esta alianza el Ministerio de Fomento Industria y Comercio (MIFIC), quien da el apoyo jurídico-legal para el proceso de registro de propiedad intelectual y marcas.

La importancia de esta investigación, radica en que si se quiere tener reconocimiento nacional e internacional por la calidad de los café producidos en Nicaragua, se deben desarrollar indicaciones geográficas y denominaciones de origen. Estos signos de calidad de café, permiten identificar los diferentes perfiles de taza de café de las distintas zonas productoras de la región norte de Nicaragua. Así mismo cabe señalar que Nicaragua ya cuenta con una ley que tiene por objeto establecer las disposiciones que regulan la protección de las marcas y otros signos distintivos.

La Denominación de Origen de un producto es el nombre de una región, de un lugar determinado, o en casos excepcionales de un país, que sirva para designar un producto agrícola o un producto alimenticio originario de dicha región cuya calidad o características se debe fundamentalmente o exclusivamente al medio geográfico con sus factores naturales y humanos, y cuya producción, transformación y elaboración se realizan en la zona geográfica delimitada (Fisac, 2010).

El propósito principal de la investigación es desarrollar una propuesta de zonificación de las calidades de café en la zona Norte de Nicaragua, e indicaciones geográficas y de ser posible la realización del primer pilotaje en denominación de origen (D.O), a través de la selección y evaluación de zonas productoras de café en la Región Norte.

El proceso de esta investigación inició en el año 2010 con la concertación de los principales actores del sector cafetalero interesado en el tema, para unificar criterios, alcances y compromisos de participación. Se logra el acceso a bases de datos de perfiles de tazas desde el año 2003 al 2010. Además se planificó la realización de dos muestreos de perfiles de taza correspondiente a las cosechas 2010-2011 y 2011-2012, caracterizando el proceso de beneficiado y levantamiento de muestras en los departamentos en estudio. 
Los resultados finales de este estudio serán sistematizados en informes, manuales técnicos así como en un atlas con los perfiles de taza de las distintas zonas productoras de café de la región norte, fortalecimiento de recurso humano local en la toma y análisis de datos, elaboración de mapas, así como manejo de bases de datos en línea de las cooperativas involucradas en la investigación.

\section{Materiales y métodos}

Para llevar a cabo el estudio de incidencia de los factores ambientales que repercuten en la calidad del café producido en el departamento de Jinotega, Matagalpa y Nueva Segovia, se utilizaron fuentes primarias con métodos cuantitativos y cualitativos para la recolección de datos y muestras de café. También se hizo una revisión bibliográfica de estudios previos realizados en calidad de café, así como datos de calidad disponibles de los eventos de taza de excelencia desde el año 2003.

La homogenización de la base de datos de perfiles de taza se realizó ajustándolos al formato SCAA (USAID 2005). Contando con los atributos de acidez, cuerpo, sabor, sabor residual, balance, taza limpia, dulzura y puntaje de catador.

Las capas de factores ambientales utilizadas son Altitud, Precipitación anual, Meses secos, Temperatura anual, Rango de temperatura diario, Punto de rocío, Radiación solar. Estas generadas con raster (grids) con resolución de 90 metros cuadrados en el caso de los mapas topográficos y una resolución de $1 \mathrm{~km}^{2}$ para los mapas de clima.

Los datos de calidad de café y condiciones ambientales de las Zonas en estudio se evaluaron estadísticamente a través de los análisis de conglomerados, componentes principales, comparación de medias y MANOVA (análisis de varianza multivariado) usando los programas InfoStat y SPSS, para poder analizar la consistencia de la calidad y la diferencia entre sitios.

Para realizar los análisis espaciales se emplearon ArcGIS 9.3, DIVA GIS y CaNaSTA, como se describen a continuación.

ArcGIS 9.3 se utilizó para realizar acciones de entrada de datos, el despliegue, consultas, análisis espacial y la impresión de mapas, tablas y gráficos. Además, para la estandarización de todas las coordenadas de fincas y los raster ambientales en un sólo sistema de georeferenciación se utilizó el datum WGS84 y grados decimales.

DIVA-GIS se utilizó para relacionar variables ambientales con sitios o puntos de muestreo. Puede leer y grabar datos en formatos estándar como los shapefiles de ESRI, de tal modo que es totalmente compatible con ArcGIS 9.3. 
CaNaSTA brinda mapas que presentan la probabilidad de éxito y el nivel de certeza de la predicción en cada localización. Este programa combina el conocimiento científico formal con información de expertos, en este caso catadores de café, para encontrar nichos ambientales de café de alta calidad, (Läderach et al., 2006).

\section{Resultados y discusión}

Como resultado de la concertación de los estudios existentes de calidad de café, se dispone de 1200 datos de perfiles de taza de la zona Norte de Nicaragua, principalmente de Nueva Segovia, Jinotega y Matagalpa (Ver tabla 1).

De las 1200 muestras de catación, 1185 han sido seleccionadas en base a su capacidad descriptiva y de modelación para alimentar las modelos espaciales y estadísticos. Este es el primer trabajo a nivel nacional que logra reunir esta cantidad de datos. Adicionalmente, se cuenta con una serie de años desde el 2003, para poder realizar un análisis de la consistencia de la calidad del café a través del tiempo, fundamentalmente a partir del año 2005. Nuevas muestras de catación de los años 2010-2011 y 2011-2012 serán incorporadas en los resultados finales de este estudio.

Tabla 1. Total de muestras por año de los datos de catación recopilados.

\begin{tabular}{|l|c|}
\hline $\begin{array}{c}\text { Ciclo de } \\
\text { Cosecha }\end{array}$ & Número de muestras \\
\hline $2002-2003$ & 60 \\
\hline $2003-2004$ & 34 \\
\hline $2004-2005$ & 32 \\
\hline $2005-2006$ & 184 \\
\hline $2006-2007$ & 488 \\
\hline $2007-2008$ & 37 \\
\hline $2010-2011$ & 365 \\
\hline TOTAL & $\mathbf{1 2 0 0}$ \\
\hline
\end{tabular}


En la figura 1 se representa la probabilidad de producir café de alta calidad en Nicaragua, indicando que las áreas del territorio Central-Norte del país se encuentran con un ambiente favorable para la producción de café de calidad.

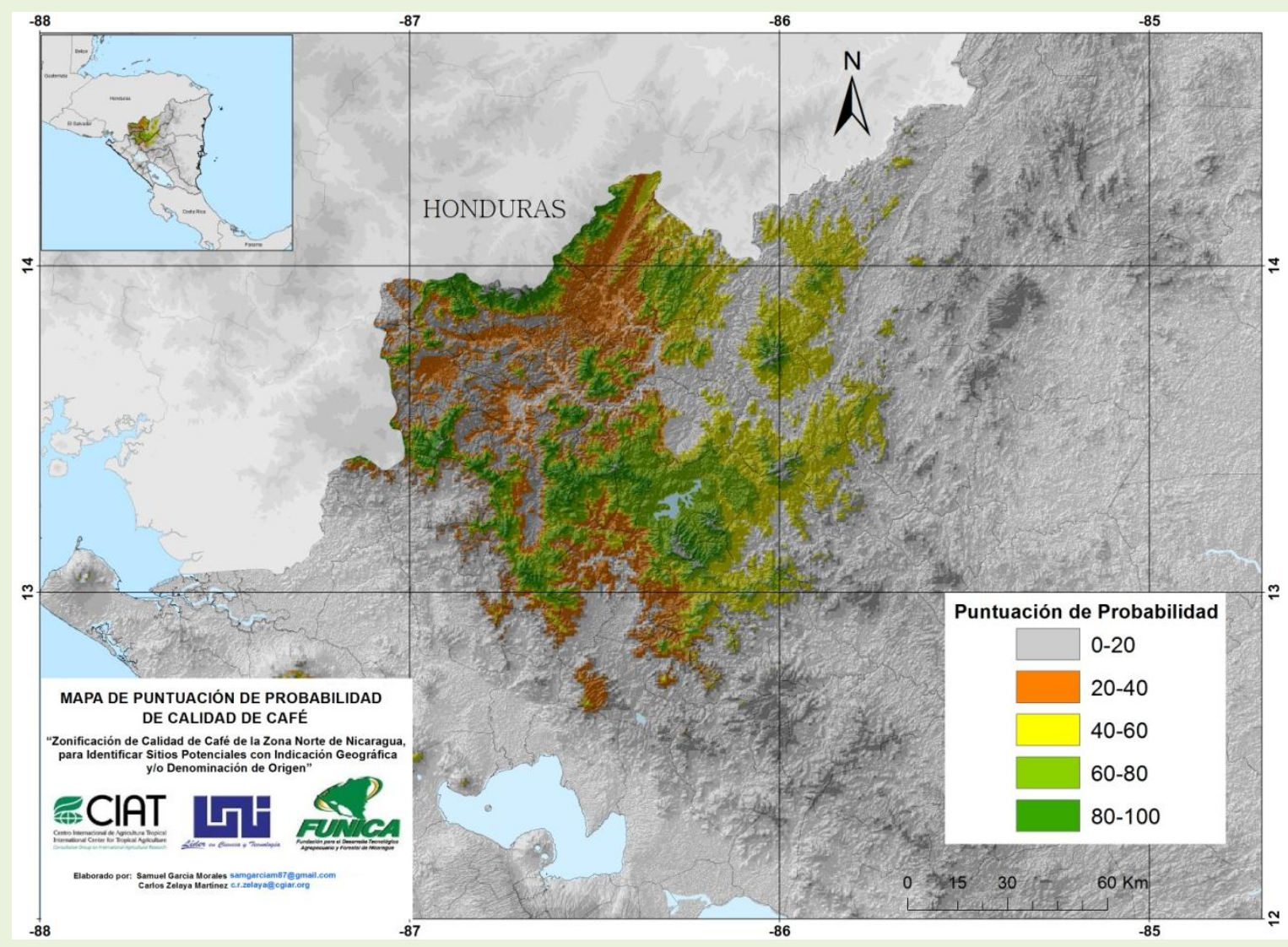

Figura 1. Puntuación de probabilidad de calidad de café en Nicaragua para identificar nichos ambientales de alta calidad.

Una de las aplicaciones de la identificación de nichos de calidad de café, es la posibilidad de predecir la calidad más probable y su posible puntuación. Los sitios potenciales para alcanzar una denominación de origen que han sido identificados son al menos cinco: Dipilto (Dipilto, Macuelizo y Mozonte), Las Sabanas (Las Sabanas, San Lucas, Pueblo Nuevo), San Juan de Rio Coco (San Juan de Rio Coco, Telpaneca) Jinotega (Jinotega, Datanli, el Diablo, Peñas Blancas) y San Rafael del Norte (San Rafael del Norte y San Sebastián de Yalí). Estos sitios mantienen consistentemente a través de los años, elevados niveles de calidad, la cual según los datos de catación es superior a los 85 puntos. Delimitaciones más finas se pueden lograr usando los datos de las fincas que están ubicadas dentro de los nichos para delimitar y caracterizar las condiciones ambientales que definen cada nicho (en proceso).

Por su extensión y sus antecedentes o reputación de calidad de café, dos zonas se vislumbran como excelentes para un pilotaje de implementación de una 
denominación de origen: Cordillera Dipilto - Jalapa y la Zona de Jinotega - Datanli, tal y como se muestra en la figura 2.

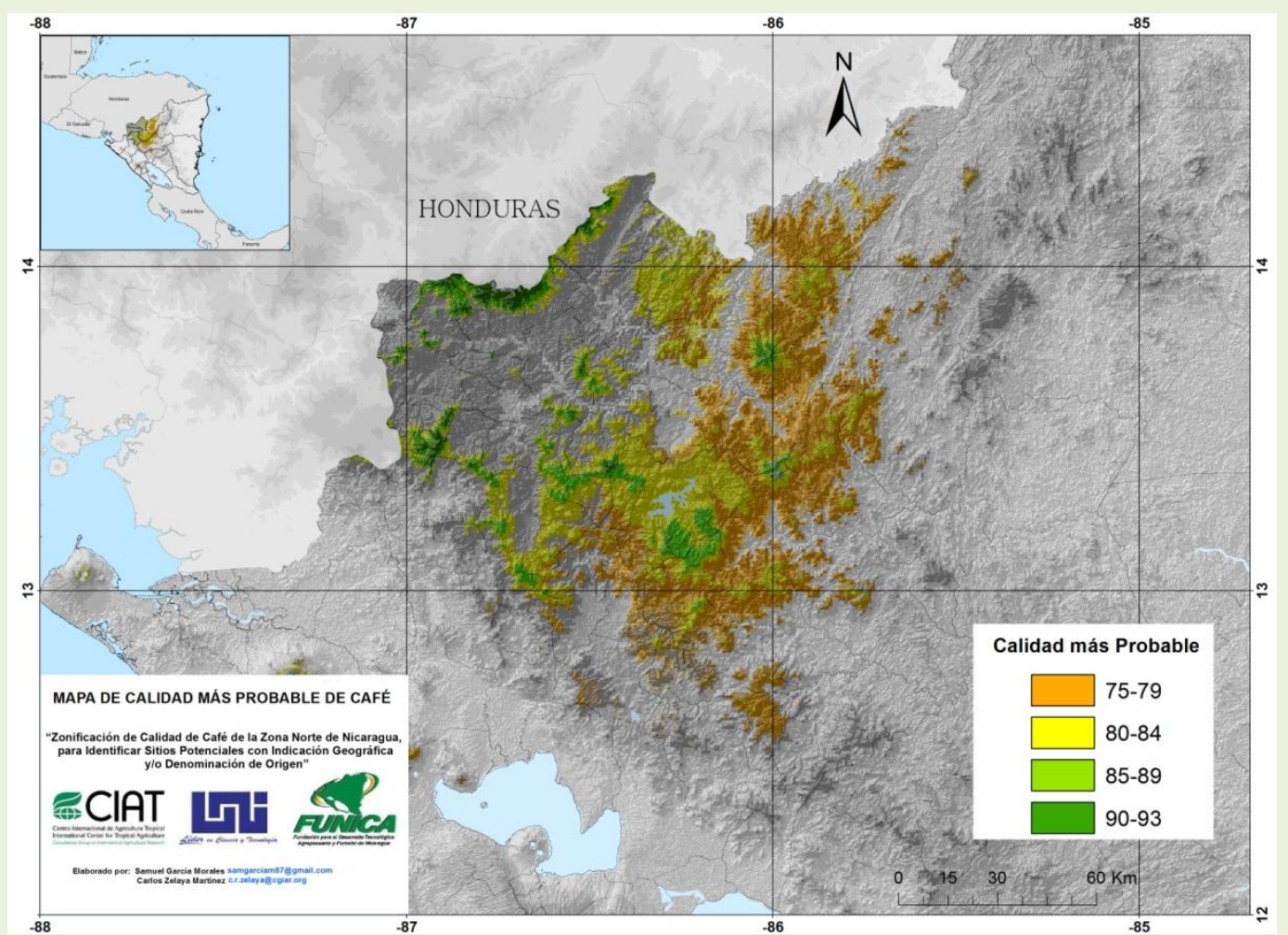

Figura 2. Calidades más probables de café en los nichos ambientales identificados

Los resultados de los análisis estadísticos de las calidades del café para el departamento de Jinotega, muestra que esta es consistente a lo largo de todos los años en que se tiene registro, con valores promedios entre 80 y 88 puntos, como se aprecia en la figura siguiente. 


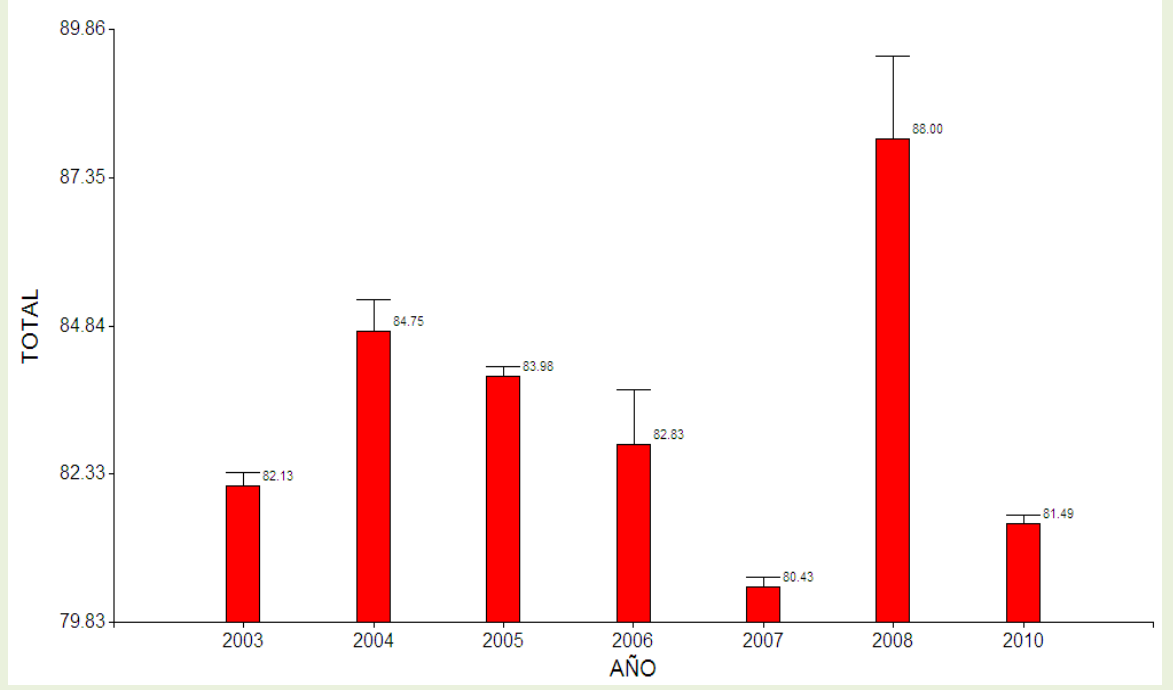

Figura 3. Análisis de datos de calidad para el departamento de Jinotega

En general el comportamiento de las calidades de café en el departamento de Nueva Segovia, también se ha mantenido consistente a lo largo de los años en que se tienen registro. Tal y como se muestra en la figura 4 , la calidad se ha mantenido con valores promedios entre 80 y 88 puntos.

DEPARTAMENTO DE NUEVA SEGOVIA

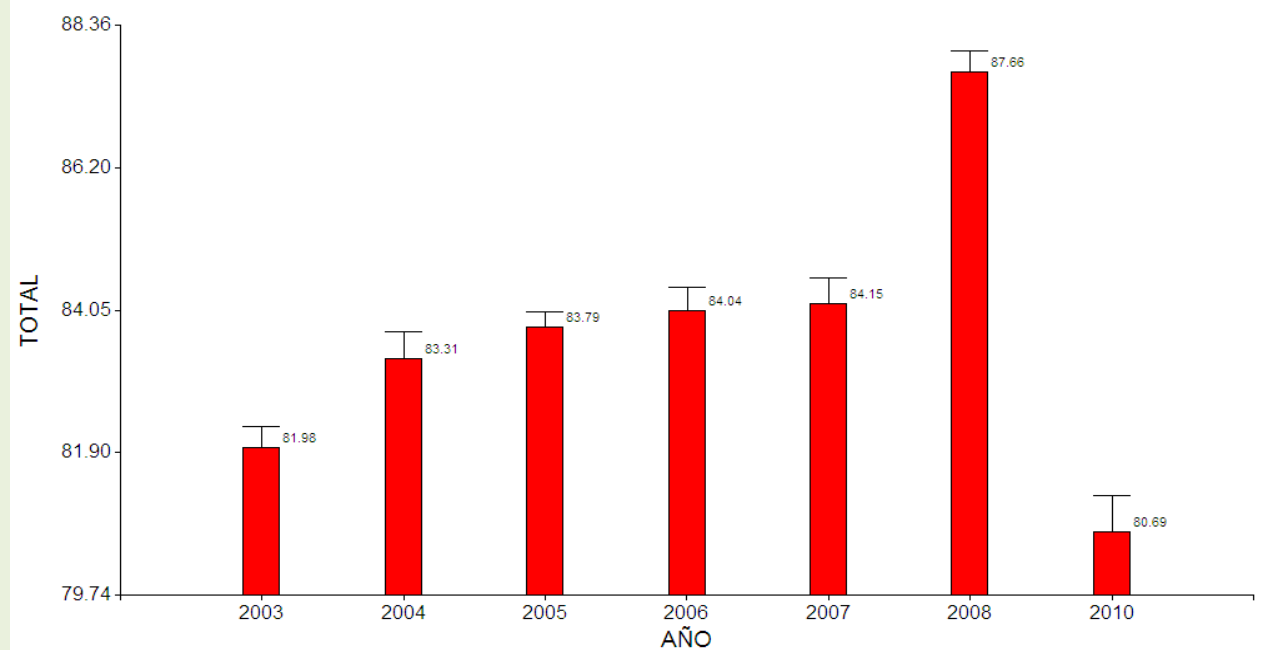

Figura 4. Análisis de datos de calidad para el departamento de Nueva Segovia 
El comportamiento de las calidades de café en el departamento de Matagalpa, muestra una creciente tendencia a mantener calidades por encima de los 80 puntos, tal y como se muestra en la siguiente figura.

DEPARTAMENTO DE MATAGALPA

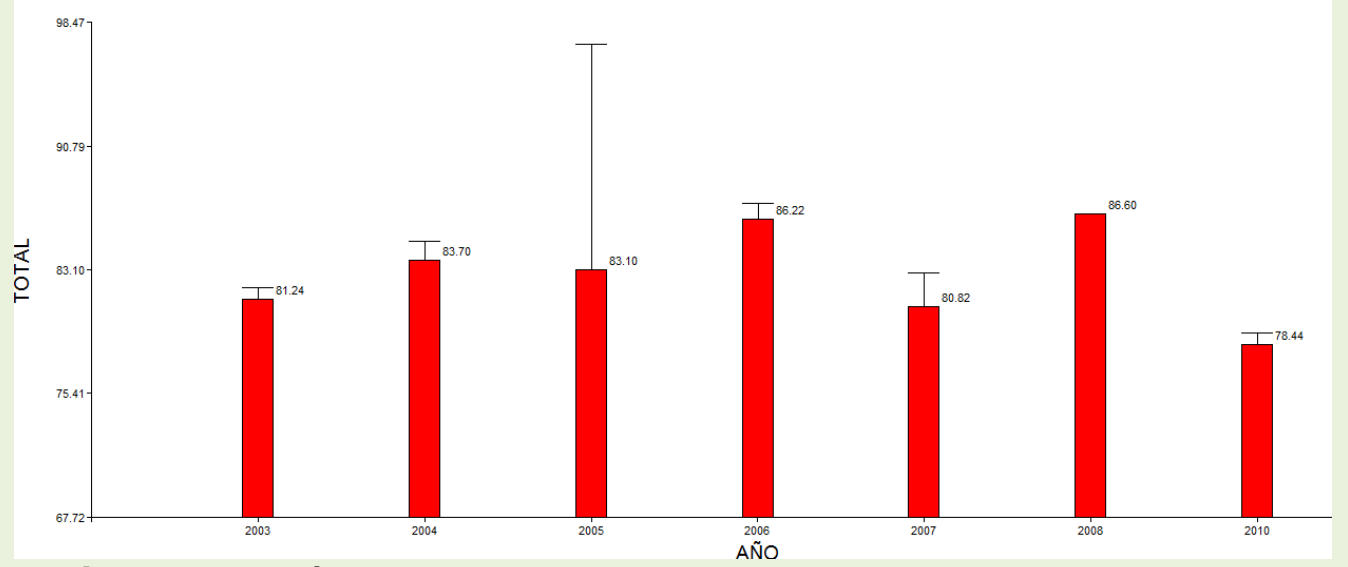

Figura 5. Análisis de datos de calidad para el departamento de Matagalpa.

Al analizar los departamentos de Nueva Segovia, Matagalpa y Jinotega desde componentes principales tal y como se muestra en la figura 6 , se puede apreciar que las características del café de Nueva Segovia son diferentes a la de los demás departamentos, teniendo una mejor tendencia de calidad este departamento.

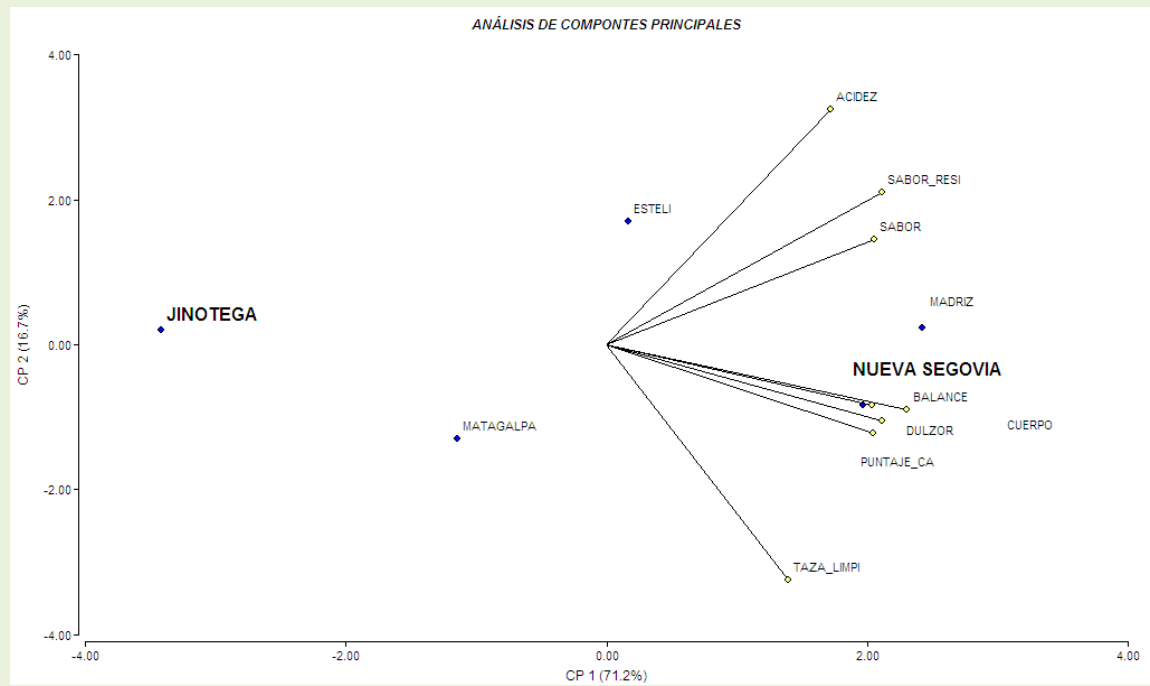

Figura 6. Análisis de componentes principales.

Al realizar el análisis por componentes principales de los atributos del café para todos los departamentos, se encontró que la mayoría tiene atributos diferentes, siendo Nueva Segovia y Madriz los que tienen una calidad muy similar. Esto se 
confirma con una prueba de análisis multivariado presentada en la Tabla 2, y análisis de conglomerado presentado en la figura 7.

Tabla 2. Análisis multivariado de atributos de café por departamento.

Análisis de la Varianza (Roy)

F.V. Estadístico $\mathrm{F}$ gl(num) gl(den) $\mathrm{p}$

DEPTO $\quad 0.6180 .54 \quad 9 \quad 1190<0.0001$

\section{Prueba Hotelling Alfa $=0.05$}

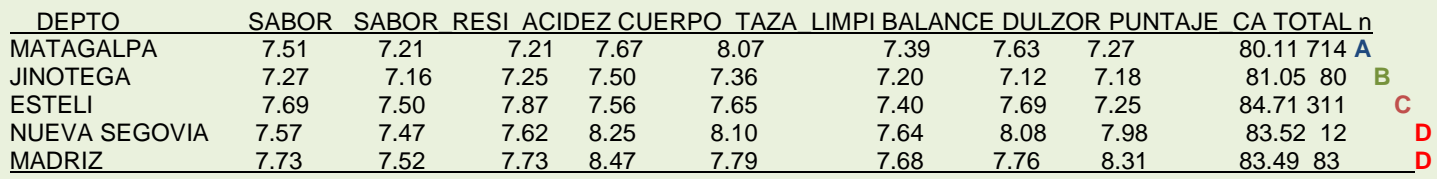

Medias con una letra común no son significativamente diferentes $(p<=0.05)$

Fuente. Elaboración a partir de base datos, UNI.

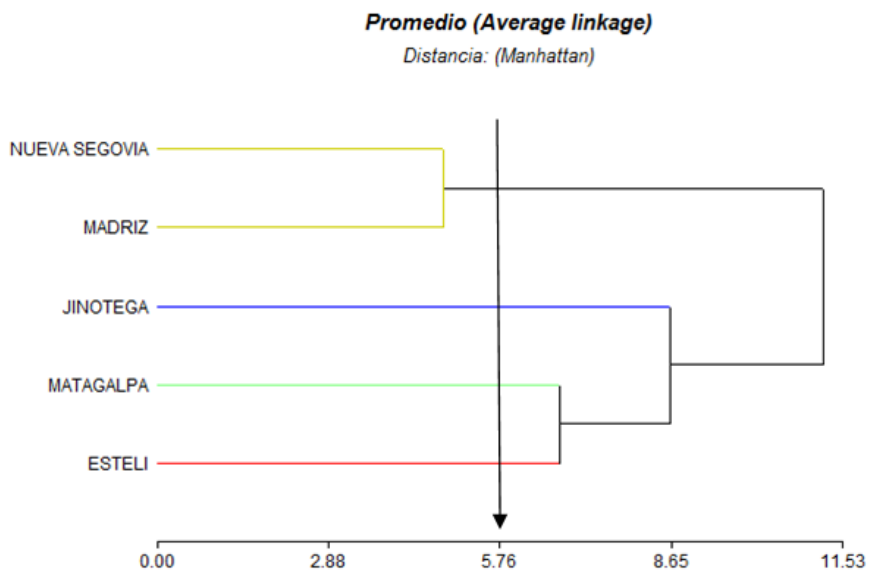

Figura 7. Análisis Conglomerados por Departamentos.

\section{Conclusiones}

La consistencia de los datos de catación son esenciales para los modelos, las condiciones ambientales influyen significativamente en la calidad del café y permiten el mapeo por atributos, Los municipios que hasta el momento mantienen cada año elevados niveles de calidad de café son al menos cinco: Dipilto (Dipilto, Macuelizo y Mozonte), Las Sabanas (Las Sabanas, San Lucas, Pueblo Nuevo), San Juan de Rio Coco (San Juan de Rio Coco, Telpaneca) Jinotega (Jinotega, Datanli, el Diablo, Peñas Blancas) y San Rafael del Norte (San Rafael del Norte y San Sebastián de Yalí), destacándose como sitios potenciales para alcanzar una denominación de origen. 
Los análisis confirman que hay un alto potencial para producir café de alta calidad en los sitios identificados.

El departamento de Nueva Segovia es el que presenta una tendencia hacia mejores atributos de calidad sobre Matagalpa y Jinotega, a partir de los análisis estadísticos multivariados.

Se continúa recopilando y procesando información para dar más consistencia a los resultados obtenidos y poder obtener los resultados finales, que serán reflejados en atlas digital y que serán publicados posteriormente.

\section{Referencias}

Fisac Pedrajas, Ramón. 2010. Denominación de Origen. Instituto Interamericano de Cooperación para la Agricultura (IICA), Guatemala.

Läderach, P., L. Collet, and J. Pohlan. 2005. Relationships between environmental factors and coffee cup quality in selected growing regions of Cauca, Southern Colombia. Proceedings of the III International Symposium of Coffee and Cacao, Santiago de Cuba, Cuba, 16-18 November 2005.

Läderach, P.C., L.; Oberthür, Th.; Pohlan, J. . 2005. Café especial y sus interacciones con factores de producción. . Memoria del $2^{\circ}$ diplomado sobre cafeticultura sustentable, Tuxtla Gutiérrez, Chiapas. .

Lingle, T.R. 2001. The Coffee Cuppers' Handbook - A systematic guide to the sensory evaluation of coffee's flavor. . Specialty Coffee Association of America, Third Edition. Long Beach, California.

Palacios, Marco Antonio. 2011. Leyes Relevantes Sobre Propiedad Intelectual. Secretaría de Integración Económica Centroamericana. Guatemala: SIECA \& USPTO.

USAID 2005. Normas y estándares de catación para la región de Centroamérica. Central America \& Dominican Republic Quality Coffee Program. Preparado por Chemonics International Inc y los Star Cuppers de Centroamérica. 